\title{
Third-Order Intermodulation Distortion due to Self-heating in Gold Coplanar Waveguides
}

\author{
Eduard Rocas ${ }^{1,2}$, Carlos Collado ${ }^{1,2}$, Nathan Orloff ${ }^{2}$, James C. Booth ${ }^{2}$ \\ ${ }^{1}$ Universitat Politècnica de Catalunya, Barcelona, CAT, 08034, Spain \\ ${ }^{2}$ National Institute of Standards and Technology, Boulder, CO, 80305, USA
}

\begin{abstract}
We present measurements and modeling of a selfheating mechanism responsible for third-order intermodulation distortion in coplanar waveguide transmission lines. Temperature variations, at the envelope frequency of the input signal, induce dynamic changes in the distributed resistance that, when mixed with the fundamental tones, give rise to intermodulation.
\end{abstract}

Index Terms - Coplanar waveguides, nonlinearities, electrothermal effects, intermodulation distortion.

\section{INTRODUCTION}

The existence of a self-heating mechanism that induces third-order intermodulation distortion (IMD3) is a well-known process in power amplifiers, on which successful efforts have been done on its modeling [1]-[4]. In contrast, passive microwave devices have received little attention, where few lumped models exist for discrete components such as connectors, attenuators and terminations [5]. For purposes of characterization and prediction, a nonlinear distributed model of the transmission lines with self-heating mechanism involved is required, in which the experimental observables can be related with the material properties and their temperature derivatives.

In this work, we present a distributed model to account for these nonlinearities induced by self-heating in transmission lines. The model introduces a thermal domain that simulates the heat flow along the transmission line and through the substrate, and is coupled to the electromagnetic domain.

The model was tested by use of coplanar waveguides (CPW) made of gold on sapphire, and showed good agreement with measured results on transmission lines of different lengths.

\section{UNIFIED MODEL}

Despite temperature-induced changes in the material properties, models usually, for simplicity, make use of only the steady-state ambient temperature to predict the device's performance, without taking into account the dynamic temperature fluctuations [6][7]. In fact, as presented in this work, time-dependent temperature fluctuations can lead to undesired nonlinear effects that must be consistently modeled by an electro-thermal model.

\section{A. Generation Mechanism}

A scheme showing the generation process of IMD3 due to self-heating is shown in Fig. 1, in which the two-tones test represents a useful method in nonlinear analysis. A two-tone signal $\left(f_{1}\right.$ and $\left.f_{2}\right)$ can be interpreted as a sinusoidal modulated signal at a frequency $\Delta f=f_{2}-f_{1}$ centered at $f_{0}=f_{1}+\Delta f / 2=f_{2}-\Delta f / 2$.

In the CPWs under study, dissipation is largely due to conductor losses for a substrate with negligible loss, such as sapphire. In such a case, the quadratic relation between instantaneous dissipated power and current implies that heat fluctuations are generated at frequencies $\Delta f, 2 \mathrm{f}_{1}, \mathrm{f}_{1}+\mathrm{f}_{2}$ and $2 \mathrm{f}_{2}$. However, the relation between dissipation $\left(\mathrm{P}_{\mathrm{d}}(\mathrm{f})\right)$ and temperature $(T(f))[8]$,

$$
T(f)=Z_{t h}(f) P_{d}(f),
$$

states that only variations at the envelope frequency $\Delta f$ will produce substantial temperature changes due to the low-pass filter behavior of the thermal impedance $Z_{t h}(f)$ that is related to the slow nature of thermal dynamics [8].

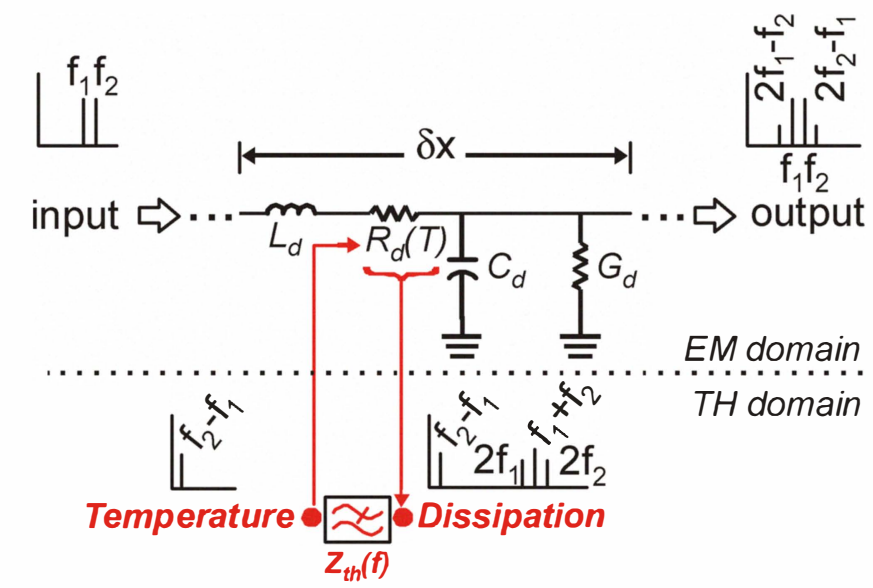

Fig. 1. Process by which IMD3 is generated in a transmission line. Resistive losses are responsible for temperature fluctuations that in turn change the metal resistivity, giving rise to a dynamically generated nonlinearity.

As seen in Fig. 1, temperature fluctuations change the metal resistivity $\rho(T)$ and, therefore, the distributed resistance $R_{d}(T)$ of the line. In fact, the temperature-induced changes can also be treated as an amplitude modulation of the input signals $f_{l}$ and $f_{2}$ by a modulating signal at frequency $\Delta \mathrm{f}$, which gives rise 
to IMD3 at $2 \mathrm{f}_{1}-\mathrm{f}_{2}$ and $2 \mathrm{f}_{2}-\mathrm{f}_{1}$. So, in such a situation, measurements of IMD3 while sweeping the tones spacing will lead to a low-pass filter shape [5] that could be used to deembed the thermal impedance if an accurate model is used:

$$
P_{I M D 3} \propto\left|Z_{t h}(\Delta f)\right|^{2}
$$

\section{B. Model Implementation}

A consistent model to account for the aforementioned process needs the interaction of an electromagnetic and a thermal domain. Then, we build the electromagnetic domain as a cascade of sections of a certain length $\Delta x$, of lumped elements $R_{d, \Delta_{x}}(T)=R_{d}(T) \cdot \Delta x, L_{d, \Delta_{x}}=L_{d} \cdot \Delta x, G_{d, \Delta_{x}}=G_{d} \cdot \Delta x, C_{d, \Delta_{x}}=$ $C_{d} \cdot \Delta x$, where $R_{d}(T), L_{d}, G_{d}$, and $C_{d}$ are the distributed parameters of the coplanar waveguide. For very thin conductor strips as used in our experiments, the distributed resistance changes with temperature as follows: $R_{d}(T)=R_{d, 0}(1+$ $\alpha T)$, where $\alpha$ is the temperature coefficient of resistivity.

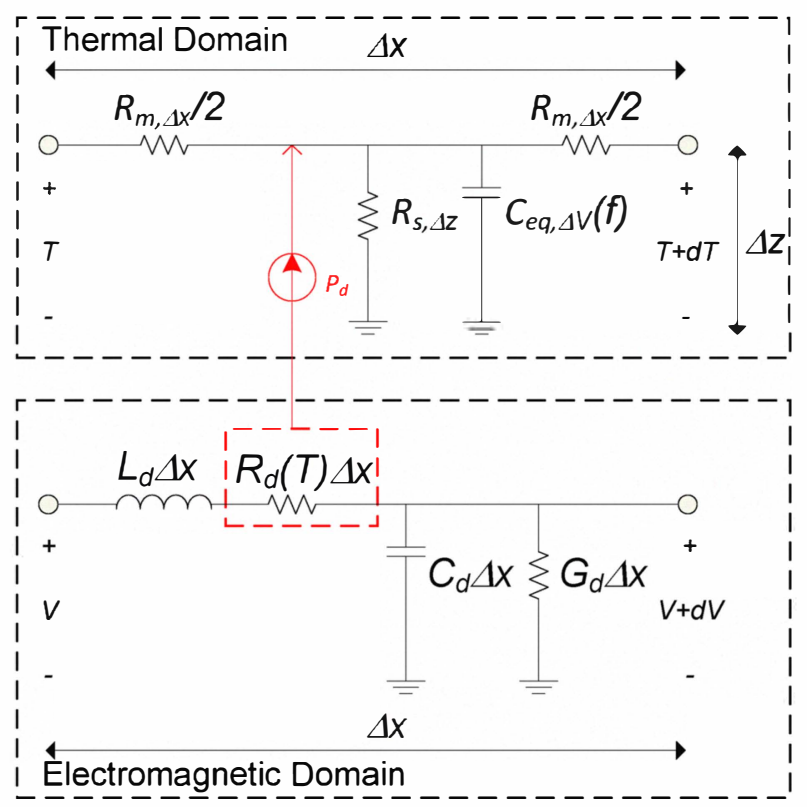

Fig. 2. Implementation of a section of transmission line in both electromagnetic and thermal domains.

On the other hand, thermal modeling of the heat flow along the transmission line and through the substrate is implemented by means of the thermal resistance $R_{t h}=\Delta_{t} / k_{t h}$ and the volumetric heat capacity $C_{t h}=\varsigma C_{p}$, that can be separately defined for the metal and the substrate [8] (in the previous expressions, $\mathrm{k}_{\mathrm{th}}, \varsigma$ and $\mathrm{C}_{\mathrm{p}}$ are the thermal conductivity, density, and heat capacity of the material, respectively, and $\Delta_{u}$ is a length increment in the direction of the heat propagation that can either be $\Delta x$ along the line or $\Delta z$ through the substrate (see Fig. 2)). So, with the above-mentioned thermal distributed parameters, each section of transmission line can be thermally modeled along the metal and through the substrate with a series resistance and shunt capacitance in the respective directions. In fact, the shunt capacitances can be combined in parallel with $\mathrm{C}_{\mathrm{eq}, \Delta \mathrm{v}}(\mathrm{f})$ to represent an equivalent overall heat capacity of the metal and substrate $\Delta \mathrm{V}$ in volume (Fig. 2).

In Fig. 2, the horizontal axis corresponds to the direction along the line, while the vertical axis is perpendicular to the wafer. So the thermal domain model presented is distributed along the horizontal axis but concentrated on the vertical axis. This means that $\Delta \mathrm{x}$ represents a section of the line and $\Delta \mathrm{z}$ is the total substrate thickness. The latter means that the $3 \mathrm{D}$ heat dissipation process has to be consistently modeled by use of a 2D model, even when the heat diffusion area on the XY plane changes as a function of frequency [8]. Such a frequencydependent lateral diffusion area can be taken into account by use of a frequency-dependent equivalent heat capacity $\mathrm{C}_{\mathrm{eq}, \Delta \mathrm{v}}(\mathrm{f})$, similar to that used in [5]. Moreover, the fact that a CPW consists of a center conductor between two ground planes implies that an equivalent width $\mathrm{W}_{\text {eq }}$ in terms of dissipation (usually related to the physical width by a geometrical factor [8]), has to be considered if a 2D model is used.

In Figure 2, $\mathrm{R}_{\mathrm{m}, \Delta_{\mathrm{x}}}=\mathrm{R}_{\mathrm{m}, \mathrm{th}} /\left(\mathrm{W}_{\mathrm{eq}} \mathrm{t}_{\mathrm{m}}\right)$ and $\mathrm{R}_{\mathrm{s}, \Delta_{\mathrm{z}}}=\mathrm{R}_{\mathrm{s}, \mathrm{th}} /\left(\mathrm{W}_{\mathrm{eq}} \Delta \mathrm{x}\right)$ are the thermal resistances for a section of metal and substrate respectively, where $t_{m}$ is the metal thickness. Heat radiation and convection are considered negligible, as well as heat flow through the probes, which are modeled using high value resistances at the terminations of the thermal domain.

Finally, dissipation in the electromagnetic domain is coupled to the thermal domain as a current source (see Fig. 2). This makes use of the fact that, in such a thermal transmission line, current and voltage are analogous to heat and temperature, respectively [8].

\section{MEASUREMENTS AND RESULTS}

Linear and nonlinear characterization of two coplanar waveguides was carried out at room temperature for purposes of model validation.

\section{A. Test Wafer and Linear Measurements}

Two $480 \mathrm{~nm}$-thick gold CPWs, with the same geometry except for different length $1_{A}=4.200 \mathrm{~mm}$ and $1_{B}=9.933 \mathrm{~mm}$, were fabricated using photolithographic techniques on a 430 $\mu \mathrm{m}$-thick sapphire substrate. A $20 \mathrm{~nm}$-thick titanium intermediate layer was used for better adhesion between gold and substrate. The center conductor, ground planes and gap widths are $30 \mu \mathrm{m}, 200 \mu \mathrm{m}$ and $15 \mu \mathrm{m}$, respectively.

A procedure of multi-line TRL calibrations and impedance comparison was applied to extract the distributed parameters of the CPWs. Details on these extraction technique can be found in [9]. Extracted resistance and inductance per unit length from measurements is shown in Fig. 3. The extracted capacitance per unit length is $C_{d}=162 \mathrm{pF} / \mathrm{m}$, and $G_{d}$ is negligible. 


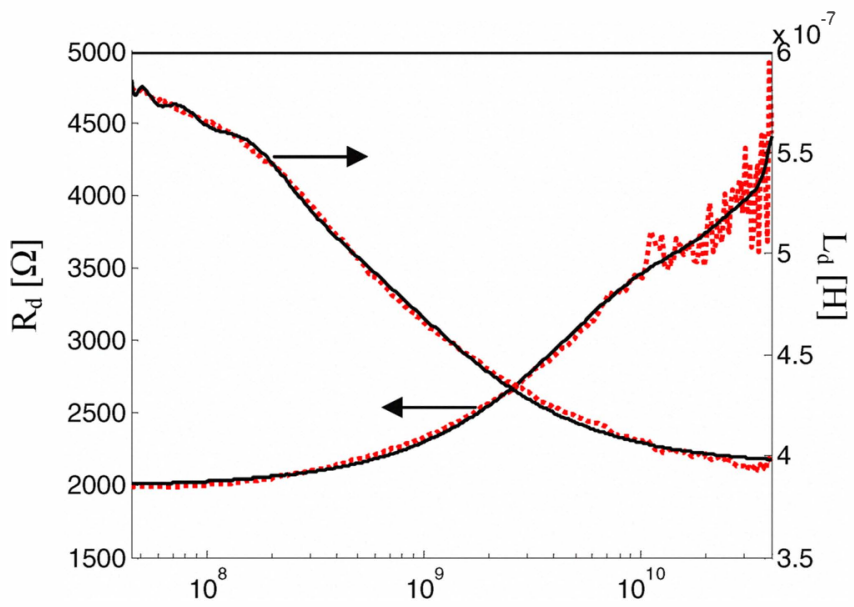

Fig. 3. Resistance and inductance per unit length as extracted from measurements (dashed lines) and a polynomial fit (solid lines).

Figure 3 also shows the polynomial fit for $R_{d}$ and $L_{d}$, which smoothes the data from measurements and is used in the circuit model.

\section{B. Nonlinear Measurements}

As previously noted, the dependence of the intermodulation distortion level on the envelope frequency of the input signal is probably the convincing evidence for self-heating induced nonlinearities. To evaluate this and to validate the model with measurements, IMD3 has been measured on both CPWs for a wide range of tone spacings. The upper tone was set at $f_{2}=6$ $\mathrm{GHz}$, while the lower tone $\mathrm{f}_{1}=\mathrm{f}_{2}-\Delta \mathrm{f}$ varied so that $\Delta \mathrm{f}$ ranges $\Delta \mathrm{f}=2 \mathrm{~Hz}$ to $\Delta \mathrm{f}=1 \mathrm{GHz}$ at an input power of $20 \mathrm{dBm}$ per tone.

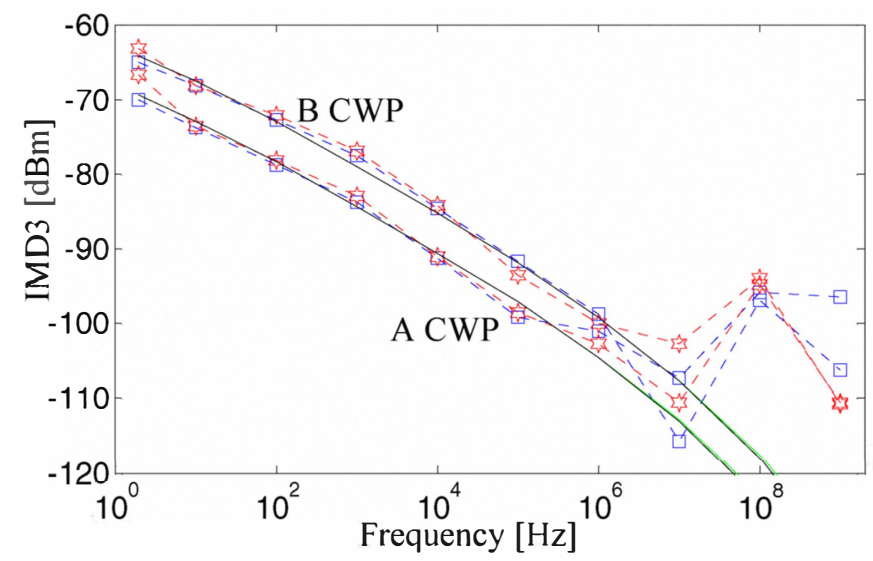

Fig. 4. Measurements $\left(2 \mathrm{f}_{1}-\mathrm{f}_{2}\right.$ and $2 \mathrm{f}_{2}-\mathrm{f}_{1}$ are squares and circles respectively) and simulations (solid lines overlapped) of the generated IMD3 of the two CPWs presented in this work. The higher IMD3 level corresponds to the longer B CPW $\left(l_{B}=9.933 \mathrm{~mm}\right)$, and the lower corresponds to A CPW $\left(\mathrm{l}_{\mathrm{A}}=4.2 \mathrm{~mm}\right)$.

A special measurement setup intended to increase the measurement dynamic range at the spectrum analyzer by means of cancellation of the fundamental tones was used.
Further details on this measurement setup can be found in [9]. The measurement results can be seen in Fig. 4 in dashed lines for both CPWs A and B.

As observed from the measurements, the baseline IMD3 level of the system itself is around $-100 \mathrm{dBm}$ for the specified input power, while the measurement noise floor is at -140 $\mathrm{dBm}$. This makes the system nonlinearities predominant over the device for modulation frequencies of $1 \mathrm{MHz}$ and above.

Theoretical thermal conductivity values used in this work are $\mathrm{k}_{\mathrm{s}, \mathrm{hh}}=42 \mathrm{~W} \cdot \mathrm{m}^{-1} \cdot \mathrm{K}^{-1}$ and $\mathrm{k}_{\mathrm{m}, \mathrm{h}}=318 \mathrm{~W} \cdot \mathrm{m}^{-1} \cdot \mathrm{K}^{-1}$ for sapphire and gold, respectively [8]. A temperature coefficient of resistivity for gold of $\alpha=0.0037 \mathrm{~K}^{-1}$ is also used for both lines [8].

Equation (2) states that the measured intermodulation level is proportional to the square of the thermal impedance, and this is used next to extract the equivalent heat capacity $\mathrm{C}_{\mathrm{eq}, \Delta v}(\mathrm{f})$ as a function of frequency, implemented as $\mathrm{C}_{\mathrm{eq}, \mathrm{v}}(\mathrm{f})=$ $\mathrm{C}_{\mathrm{d}, \mathrm{h}}(\mathrm{f}) \cdot \mathrm{W}_{\mathrm{eq}} \cdot \mathrm{z}$.

The procedure to extract $\mathrm{C}_{\mathrm{eq}, \Delta \mathrm{V}}(\mathrm{f})$ from the measured results by means of (2) starts by obtaining the magnitude of the unscaled thermal impedance $Z_{\text {th.u }}(f)$. Then, $\quad\left|Z_{\text {th.u }}(f)\right|$ is extrapolated to obtain the theoretical DC value of the thermal resistance, given by $R_{s, \Delta z}$. Finally, $C_{e q, \Delta V}(f)$ is obtained from the following relation:

$$
C_{e q, \Delta V}(f)=\sqrt{\frac{\frac{1}{\left|Z_{t h, u}(f)\right|}-\frac{1}{R_{s, \Delta z}^{2}}}{f^{2}}} .
$$

By means of the procedure above, an equivalent heat capacity can be described using a polynomial in log-log scale:

$$
C_{d, t h}(f)=10^{C_{t h, 0}+C_{t h, 1} \log (f)+C_{t h, 2} \log (f)^{2}},
$$

in which $\mathrm{C}_{\mathrm{th}, 0}=3.94, \mathrm{C}_{\mathrm{th}, 1}=-0.784$ and $\mathrm{C}_{\mathrm{th}, 2}=0.0186$ are used in the model to simulate the results shown in Fig. 4. A low-order polynomial is used to keep the tendency of the thermal impedance at frequencies above those used for the fitting.

As can be seen in Fig. 4, the agreement between measurements and modeling is very good for both lines using $\mathrm{W}_{\mathrm{eq}}=700 \mu \mathrm{m}$. Only a small deviation is observed at the modulation frequency of $2 \mathrm{~Hz}$, which could be an indication of the probes effect. 


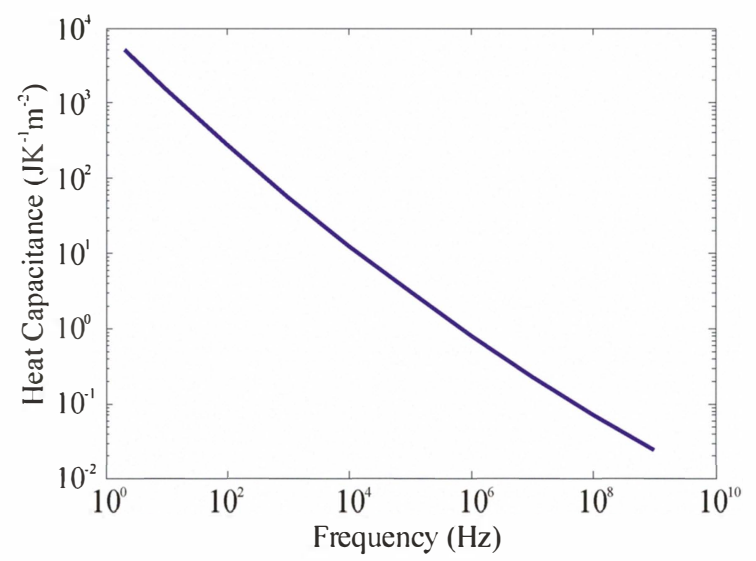

Fig. 5. Extracted heat capacitance $\mathrm{C}_{\mathrm{d} . \mathrm{th}}(\mathrm{f})$.

\section{DISCUSSION}

Further research could be performed to relate the extracted frequency-dependent equivalent heat capacity to the heat diffusion area. This may result in a better description of the $3 \mathrm{D}$ dissipation process.

The model presented in this work is useful not only for use in nonlinear prediction but also also for simulating the steadystate temperature distribution along the transmission lines. Figure 6 shows the current and temperature distribution for CPW B $\left(l_{\mathrm{B}}=9.933 \mathrm{~mm}\right)$ with a clear cause-effect relation between them. In fact, the nonlinear generation mechanism presented has some similarities with the $3 \omega$ technique [10], in which the generated third harmonic is used to extract the thermal properties of the substrate at low frequencies.

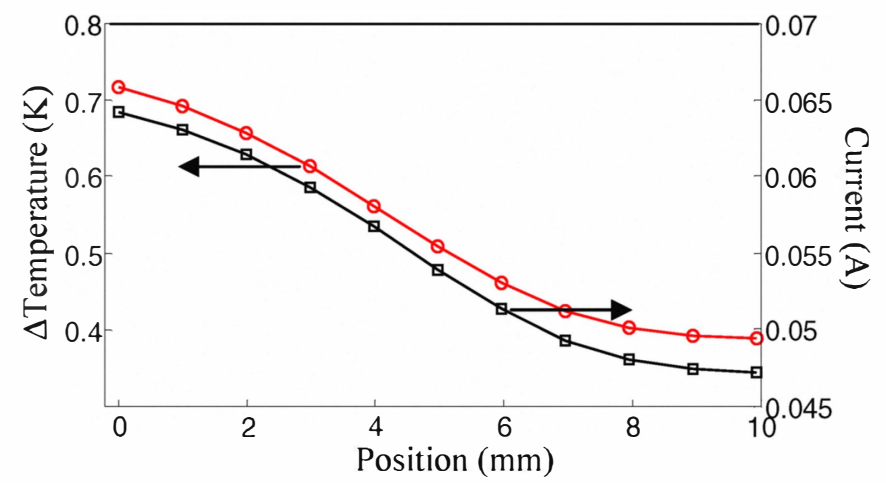

Fig. 6. Simulation of the steady state temperature increment distribution (circles) and current distribution (squares) along CPW B $\left(l_{B}=9.933 \mathrm{~mm}\right)$.

Future research will consider metals other than gold, with higher and lower temperature coefficients of resistivity. A thicker metal layer should also be evaluated to check the validity of the model. Substrates with better and poorer thermal conductivities could also be addressed for a better understanding of how the self-heating impacts the IMD3 level.

\section{ACKNOWLEDGEMENT}

This work was partially supported by the Spanish Government (CICYT) under Grant TEC-2006-13248-C0402/TCM. E.R. thanks the support from BES-2007-16775. C.C thanks the support from Generalitat de Catalunya, grant 2008BE2-00196. The authors thank David Novotny for his help in performing this work.

\section{REFERENCES}

[1] A. E. Parker and J. G. Rathmell, "Self-heating process in microwave transistors", in URSI Commission C Appl. Radio Sci. Workshop, P. Wilkinson, Ed., Hobart, Australia, 2004, pp. 1-8

[2] J. Vuolevi, T. Rahkonen, "Analysis of third-order intermodulation distortion in common-emitter BJT and HBT amplifiers," IEEE Trans. on Circuits and Systems II: Analog and Digital Signal Processing, vol.50, no.12, pp. 994-1001, Dec. 2003

[3] V. Camarchia, F. Cappelluti, M. Pirola, S. D. Guerrieri, G. Ghione, "Self-consistent electrothermal modeling of class A, $\mathrm{AB}$, and $\mathrm{B}$ power GaN HEMTs under modulated RF excitation", IEEE Trans. on Microwave Theory and Techniques, vol. 55, pp. 1824-1831, 2007.

[4] J. Vuolevi, T. Rahkonen, J. Manninen, "Measurement technique for characterizing memory effects in RF power amplifiers", IEEE Trans. on Microwave Theory and Techniques, vol. 49, no 8, August 2001, pp. 1383-1389

[5] J. R. Wilkerson, K. G. Gard, A. G. Schuchinsky, M. B. Steer, "Electro-thermal theory of intermodulation distortion in lossy microwave components", IEEE Trans. on Microwave Theory and Techniques, vol. 56, no. 12, Part 1, pp. 2717-2725, Dec. 2008.

[6] N. B. Hassine, D. Mercier, P. Renaux, D. Bloch, G. Parat, B. Ivira, P. Waltz, C. Chappaz, R. Fillit, S. Basrour, "Self heating under RF power in BAW SMR and its predictive 1D thermal model", Frequency Control Symposium, 2009 Joint with the 22nd European Frequency and Time forum. IEEE International, vol., no., April 2009, pp.237-240.

[7] B. Ivira, P. Benech, R. Fillit, F. Ndagijimana, P. Ancey, G. Parat, "Modeling for temperature compensation and temperature characterizations of $\mathrm{BAW}$ resonators at $\mathrm{GHz}$ frequencies", IEEE Trans. on Ultrasonics, Ferroelectrics, and Frequency Control, vol. 55, no. 2, pp. 421-430, Feb. 2008

[8] F. P. Incropera and D. P. de Witt, Fundamentals of Heat and Mass Transfer, 5th ed., J. Wiley \& Sons N.Y., 2002.

[9] J. Mateu, J. C. Booth, S. A. Schima, "Frequency tuning and spurious signal generation at microwave frequencies in ferroelectric $\mathrm{SrTiO}_{3}$ thin-film transmission lines", IEEE Trans. on Microwave Theory and Techniques, vol. 55, no. 2, pp. 392396, Feb. 2007.

[10] D. G. Cahill, "Thermal conductivity measurement from 30 to $750 \mathrm{~K}$ : The $3 \omega$ method", Review of Scientific Instruments, vol. 61, pp. 802-808, 1990. 